\title{
Fournier's Gangrene: Experience with Two Severe Cases
}

\author{
Afoko Akisibadek Alekz¹, Hoyte Williams², Afoko Vivian³ ${ }^{3}$ Yussif Adams ${ }^{*}$ \\ ${ }^{1}$ Department of Surgery, School of Medicine, University for Development Studies, Tamale, Ghana \\ ${ }^{2}$ Department of Surgery, School of Medical Sciences, Kwame Nkrumah University of Science and Technology, Kumasi, Ghana \\ ${ }^{3}$ Department of Paediatric Nursing, School of Nursing and Midwifery, University for Development Studies, Tamale, Ghana \\ ${ }^{4}$ Department of Biomedical Laboratory Science, School of Allied Health Sciences, University for Development Studies, Tamale, Ghana \\ Email: *adamsyussif@uds.edu.gh
}

How to cite this paper: Alekz, A.A., Williams, H., Vivian, A. and Adams, Y. (2021) Fournier's Gangrene: Experience with Two Severe Cases. Open Journal of Urology, 11, 273-281.

https://doi.org/10.4236/oju.2021.117025

Received: June 12, 2021

Accepted: July 19, 2021

Published: July 22, 2021

Copyright (c) 2021 by author(s) and Scientific Research Publishing Inc. This work is licensed under the Creative Commons Attribution International License (CC BY 4.0).

http://creativecommons.org/licenses/by/4.0/

\begin{abstract}
Fournier's Gangrene is a formidable rare disease characterized by high mortality rates despite optimal medical and surgical management. It is an acute surgical emergency and requires a high degree of suspicion. The mainstay of treatment is swift open drainage and early aggressive surgical debridement of all necrotic tissue, followed by targeted antibiotic therapy. The authors report on two patients who were admitted to the surgical ward. Both presented with initial perianal sepsis complicated by painful swollen scrotum with rapid progression of gangrene of the scrotal skin and subcutaneous tissue, extensive cellulitis of the perineum, and in one patient crepitation of the anterior abdominal wall. A diagnosis of Fournier's Gangrene was made and they were both managed by prompt resuscitation, broad-spectrum antibiotics, extensive debridement of all necrotic tissue in theatre, daily wound dressing, and repeated neurectomy on the ward. One of the patients had a colostomy done and the other a cystostomy to divert feces and relieve chronic urinary retention respectively. These patients were successfully treated despite the severity of their conditions which was complicated by severe sepsis in the face of limited diagnostic capabilities and resources, using a multidisciplinary approach and basic clinical monitoring as a guide.
\end{abstract}

\section{Keywords}

Fournier's Gangrene, Surgical Debridement, Necrotic Tissue, Sepsis, Antibiotic Therapy

\section{Introduction}

In 1883, the French venereologist Jean Alfred Fournier described a series in which 
5 previously healthy young men suffered from rapidly progressive gangrene of the penis and scrotum without apparent cause. This condition is now known as Fournier's gangrene (FG) [1].

Fournier's gangrene (FG) is a rare, synergistic, fulminant form of necrotizing fasciitis involving the genital, perineal, and perianal regions [2], with gangrene of the overlying skin.

The incidence of the disease is higher in males with Sorensen et al., reporting an overall incidence rate of 1.6 cases per 100,000 males/year [3]. Eke [2] found a mean incidence of 97 cases per year in a review of 1726 cases in a 10 -year retrospective study.

Fournier's gangrene is characterized by high mortality rates, the reported mortality rate for FG widely varies from $20 \%-40 \%$ to as high as $75 \%-88 \%$ [1] [3] [4]. The predisposing factors believed to contribute to the development of the disease are diabetes mellitus, alcoholism, malignancies, immunosuppression, liver, and renal disease [5].

It is an acute surgical and urological emergency. The keystones of management are hemodynamic stabilization, effective antibiotic treatment, and urgent aggressive surgical debridement [5].

We present two cases of Fournier's gangrene following perianal sepsis which was successfully treated in our department despite the severity of the patient's condition in the face of limited diagnostic and monitoring capabilities.

\section{Case 1}

A 45-year-old male who had undergone a below-knee amputation of the left leg on account of a chronic ascending ulcer (No histology diagnosis available) about 4 years ago, was admitted by the urology team with severe painful swollen scrotum of 3 days duration and infected perianal ulcers of about 2 weeks associated with high-grade fever and offensive discharge. He also had a painless inability to pass urine of 2 days duration. On examination; the Patient looked moderately wasted, was pale and febrile $39.0^{\circ} \mathrm{C}$, hypotensive $(80 / 50 \mathrm{mmHg})$ tachycardic $(104$ bpm regular and bounding), and Respiratory rate was $24 \mathrm{cpm}$.

He had a grossly distended bladder, $5 \mathrm{~cm}$ above the umbilicus. Infected perianal ulcers were identified, the scrotum was grossly swollen with gangrene of the anterior part of the skin and cellulitis of the perineum (Figure $1(\mathrm{~A})$ and Figure 1(B)).

Hemoglobin was $8.6 \mathrm{~g} / \mathrm{dL}$, white blood cells (WBC) 18,000 u/L, Platelets 382, Sickling was Negative, Fasting blood sugar (FBS) was $6.4 \mathrm{mmol} / \mathrm{L}$ and the retroviral screen was negative.

A diagnosis of Septic Shock secondary to Fournier's Gangrene and perianal sepsis was made as well as Chronic Urinary Retention.

The patient underwent aggressive fluid resuscitation and hemodynamic support, Intravenous third-generation cephalosporin and metronidazole were commenced immediately and the patient was prepared for emergency suprapubic 


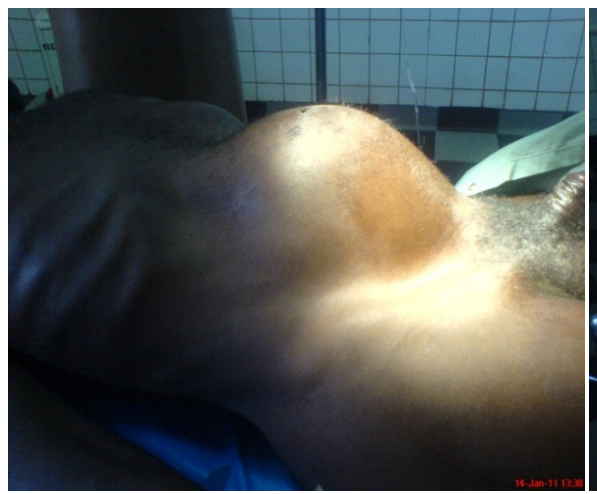

(A)

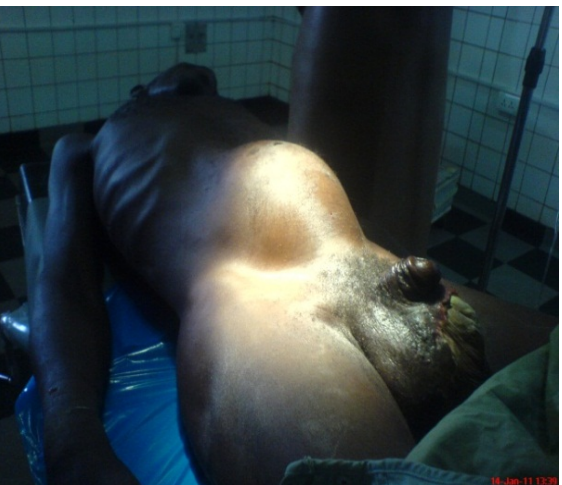

(B)

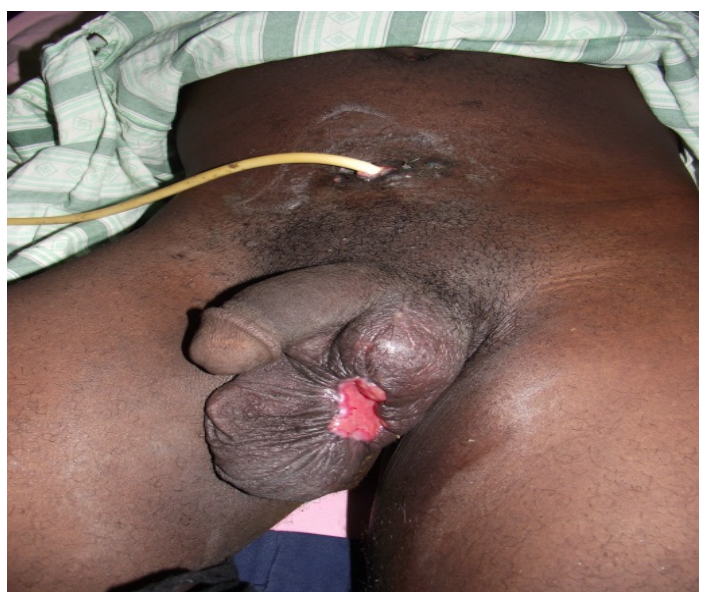

(C)

Figure 1. The picture shows Case 1-Chronic retention of urine with gross distension of bladder and gangrene of scrotal skin (A and B). The last picture (C) was taken after 6 weeks post-surgery showing a residual small ulcer which was closed in theatre.

cystostomy and debridement under Local anesthesia and conscious sedation respectively.

Intraoperatively, 2.4 litres of dark urine were drained from the bladder gradually, scrotal skin had a gangrenous patch anteriorly of about $+6 \mathrm{~cm}$, and about $0.4 \mathrm{~L}$ of purulent discharge was drained from the scrotum up to the inguinal area. Testis and Spermatic cords were intact and healthy.

Postoperatively the patient was put on intravenous Ampicillin (500 mg qid), Metronidazole (400 $\mathrm{mg}$ tid), and Gentamycin ( $80 \mathrm{mg}$ only in the evenings to reduce nephrotoxicity) for 72 hours with instructions for 6 hourly daily dressing. He underwent series of subsequent wound debridement on the ward under analgesia and the moist dressing was with normal saline-soaked gauze. Wound swab for culture yielded Staphylococcus, micro-aerophilic hemolytic Streptococcus, E. Coli, and Cl. Welchii, and sensitivity results (Augmentin, Ceftriaxone, Meropenem and Gentamicin) guided the antibiotic therapy. Patient received intravenous doses of Augmentin (1 gm bd) and Ceftriaxone (1 gm daily).

Intravenous antibiotics were converted to oral after post-op day 7, spiking of temperature resolved by post of day 5 . Glucose remained normal and healthy 
granulation tissue was observed after about 4 weeks of the wound dressing. Recovery from then onwards was progressive and uneventful.

He was discharged on the $50^{\text {th }}$ post-op day and reviewed in the outpatient department (OPD) a week later, scrotal wound was almost closed leaving a small patch that has been repaired in theatre, he can now void urine through his urethra and cystostomy has been closed (Figure 1(C)).

\section{Case 2}

A 35-year-old man with a one-year history of $3^{\text {rd }}$-degree hemorrhoids presented to the OPD with a 4-day history of perianal pain. The pain was throbbing in nature and associated with painful defecation. An impression of thrombosed hemorrhoids was made and the patient was sent to the medical ward. Severe tenderness of the perianal area with non-cooperation to examination was documented. His blood pressure (BP) was $110 / 80 \mathrm{mmHg}$, pulse 68 beats per minute, Respiration rate 16 cycles per minute, and temperature $37.0^{\circ} \mathrm{C}$.

Two days on admission, the swelling had progressed to involve the whole scrotum associated with severe pain, difficulty in micturition, and fever. Pulse was now $100 \mathrm{bpm}$, Respiratory rate was $22 \mathrm{cpm}$, and the temperature was $38.8^{\circ} \mathrm{C}$ and BP 90/60. There was crepitus over the right iliac fossa and right lumber areas of the anterior abdominal wall associated with severe tenderness. A urologist consult was sought and a diagnosis of Severe Sepsis secondary to an Ischiorectal abscess affecting the scrotum due possibly to a gas-forming bacteria was made. The patient was transferred to the surgical ward and prepared for emergency incision and drainage. Hemoglobin was $14.5 \mathrm{~g} / \mathrm{dL}$, fasting blood sugar was $6.8 \mathrm{mmol} / \mathrm{L}$, Intravenous Ciprofloxacin $200 \mathrm{mg}$ bd and Metronidazole $400 \mathrm{mg}$ tid commenced as well as fluid administration. Over the next few hours, the ischiorectal abscess ruptured with the overlying skin gangrenous and exposing the anorectum circumferentially, anal hygiene was grossly inconveniencing and the scrotal skin was rapidly becoming gangrenous. The general surgeons were involved in the plan for fecal diversion (Figure 2(A) and Figure 2(B)).

The working diagnosis was now ischiorectal abscess complicated by Fournier's Gangrene. In theatre, under general anesthesia, a double-barrel sigmoid colostomy was accomplished, and extensive necrosectomy of the scrotal skin and perineum was done evacuating the area into open drainage. The testis and spermatic cords were debrided and remained intact. Copious amounts of pus were expressed from the right lumber and right iliac fossa area via the groin. About $500 \mathrm{ml}$ of pus drained in total. The cavity was irrigated with copious amounts of Normal saline and Povidone Iodine and dressed in gauze and absorbent material. The sample was taken for culture yielded Staphylococcus, micro-aerophilic hemolytic Streptococcus, E. Coli, Cl. Welchii, and Bacteroides.

Postoperatively, 6 hourly dressing was instituted with normal saline-soaked gauze under sedation and analgesia, colostomy functioned normally, Intravenous ciprofloxacin $200 \mathrm{mg}$ bd, clindamycin $300 \mathrm{tid}$, and metronidazole $400 \mathrm{mg}$ tid 


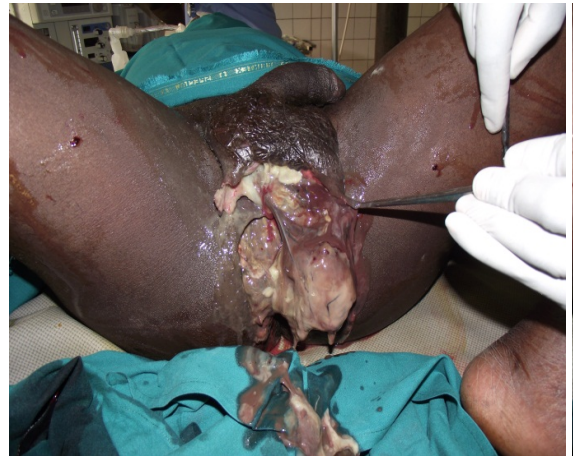

(A)

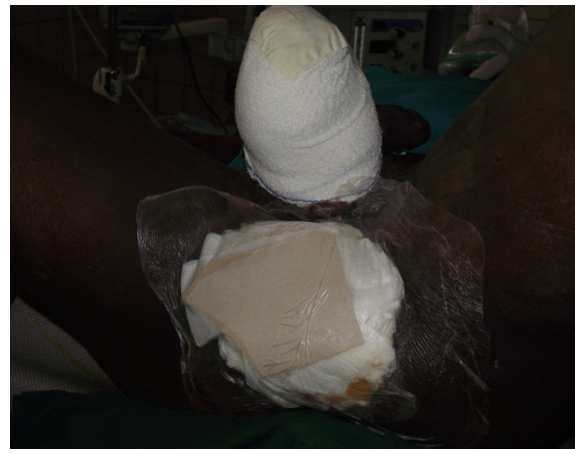

(C)

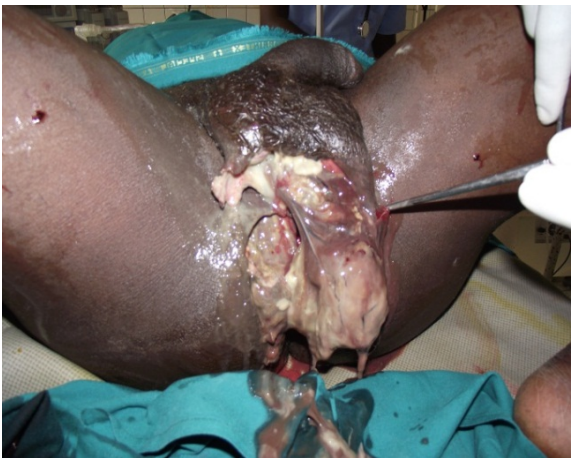

(B)

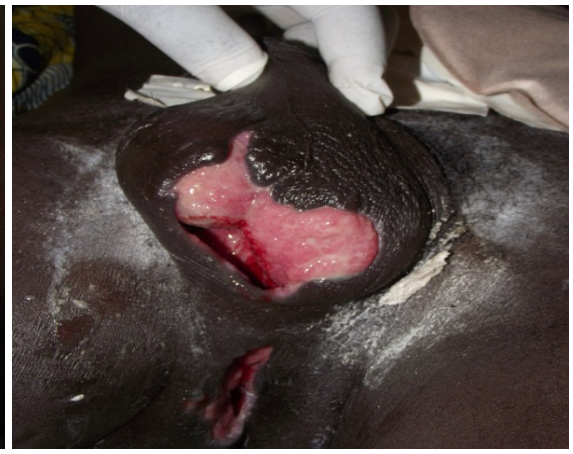

(D)

Figure 2. Pictures of Case 2-Findings before surgery showing the extent of disease (A, B and C) and 3 weeks after surgery revealing significant wound healing (D).

continued for 8 days. The patient continued to spike high temperatures associated with rigors daily for 7 days with attenuation of intensity. The broad-spectrum antibiotic response was monitored by the patient's vital signs; pulse, respiratory rate, temperature pattern blood pressure, and wound progress.

A high protein diet was encouraged, as well as ambulation. Healthy granulation tissue was observed after about 2 weeks of daily dressing, blood glucose remained normal, and the patient gradually gained weight. Hemoglobin rose to 12.0 $\mathrm{g} / \mathrm{dL}$, retroviral screen-negative. He was able to void urine through the external meatus.

Has had scrotoplasty done and the perineal ulcer healed (Figure 2(C)). Return of colostomy was carried out after 5 weeks (Figure 2(D)).

\section{Discussion}

Fournier's gangrene (FG) is the result of a highly lethal and rapidly progressive necrotizing infection of the perineal and genital fascia, with gangrene of the overlying skin [6].

The syndrome of FG is an uncommon but quite serious problem. This entity affects both men and women and at a wide age range, from neonates to the very elderly [5].

The male-to-female ratio is approximately 10:1. The lower incidence in females may be caused by better drainage of the perineal region through vaginal 
secretions. Most reported cases occur in patients aged 30 - 60 years. A literature review found only 56 pediatric cases, with $66 \%$ of those in infants younger than 3 months [1]. Our patients were 45 years and 35 years old, which is following the reported age incidence.

The disease was originally described as idiopathic, of sudden onset, and occurred in previously healthy patients [7]. In contrast to the original description, doctors today know that the disease is not limited to young or male patients, and a causative etiology is usually identified [1].

As seen now, in the majority of cases, there is an underlying problem [7] such as, genitourinary infections, Anorectal sepsis, and traumatic infections which are the most common causes of Fournier's gangrene [6].

With regards to the genitourinary tract, urethral strictures and transurethral instrumentation are the most frequent aetiologies; other causes include surgery of the penis and scrotum, transrectal prostate biopsy, urethral calculi, bladder cancer infiltrating the urethra, and phlebitis of dorsal penis vein [5]. Anorectal sources of infection include ischiorectal, perianal, and intersphincteric abscesses, especially those inadequately treated [8]. Diverticular perforation, carcinoma of the sigmoid colon and rectum, perforated acute appendicitis, internal hemorrhoids ligated with rubber bands, and anal dilatation, have also been reported in the etiology of FG [5].

In women, septic abortions, vulvar or Bartholin gland abscesses, hysterectomy, and episiotomy are documented sources.

In men, anal intercourse may increase the risk of perineal infection, either from blunt trauma to the area or by the spread of rectally carried microbes.

In children, circumcision, strangulated inguinal hernia, omphalitis, insect bites, trauma, urethral instrumentation, perirectal abscesses, systemic infections, and burns have led to the disease [1].

Inability to practice adequate perineal hygiene or the presence of chronically indwelling catheters, such as in paraplegic patients, poses an increased risk [1].

In our report, perianal Sepsis was found to be the initiating cause of the Fournier's gangrene in both patients.

It is a polymicrobial infection caused by the synergistic, opportunistic infection of both aerobic and anaerobic organisms including; Staphylococcus, micro-aerophilic hemolytic Streptococcus, E. Coli, Fusobacterium, Cl. welchii, and Bacteroides from the patient [7]. The infection arises from bacteria inoculation in the perineal area. This procedure can be facilitated by any condition that results in impairment in the immune system [5]. Wound cultures from patients with Fournier gangrene reveal an average of 4 isolates per case [1].

The bacteria involved act synergistically, via collagenases, hyaluronidases, and other enzymes to invade and destroy fascial planes. Ultimately, an obliterative endarteritis develops, and the ensuing cutaneous and subcutaneous vascular necrosis leads to localized ischemia and further bacterial proliferation. Rates of fascial destruction as high as $2-3 \mathrm{~cm} / \mathrm{h}$ have been described [1]. 
The cutaneous manifestations of Fournier's gangrene are mere "the tip of the iceberg" because the infection spreads aggressively along recognized fascial planes [6].

The most common clinical features are perianal pain and swelling if the anorectal area is the portal of entry, whereas urinary retention, testicular, or scrotal pain is present if the infection launches from the genitourinary tract [8]. Other systemic manifestations such as fever, tachycardia, hypotension, electrolyte imbalances, and hyperglycemia may also be present [5].

In our case, both patients were admitted with severe perianal pain, had scrotal swelling and pain. One of them had anterior abdominal wall crepitus which was indicative of Clostridium species involvement. Both had a fever, tachycardia, and low blood pressure indicative of severe sepsis and were resuscitated with fluid therapy.

Various studies serve as diagnostic adjuncts; however, a definitive diagnosis is made during the initial surgical wound exploration [9].

Once a diagnosis of FG has been established, the central principles of management are aggressive hemodynamic stabilization, parenteral broad-spectrum antibiotics which will be either changed or continued according to the culture findings and nutritional support. However, the most important aspect of treatment to ensure a successful outcome is urgent and extensive surgical debridement [2]. All frankly necrotic tissue and those with doubtful viability should be carefully debrided and excised. Multiple surgical procedures may be necessary to bring the infection under control. High mortality rates may stem from multiple comorbidities but unequivocally are due to delayed diagnosis and surgical treatment. In the majority of instances, the testes, glans penis, bladder, and rectum are spared destruction because of their separate blood supplies [5] [6] [9].

Targeted antibiotic therapy is commenced based on culture results. In our case, we continued with broad-spectrum antibiotics based on culture and sensitivity results and using clinical examinations such as temperature pattern, pulse, respiration, and general well-being of the patient as a guide.

In some studies, patients underwent orchiectomy when severe infection in the peritesticular tissues was observed intraoperatively [5]. Both patients in this report had their testes and spermatic cords intact and not completely exposed.

Diversion of the fecal and urinary streams may not always be necessary but should always be considered on a case-by-case basis [6].

We involved the general surgeons who performed a diverting colostomy in one of our patients since the gangrene extended to the perianal area and anal sphincter was involved as such fecal contamination was expected. The other patient had a suprapubic cystostomy on account of the failure of catheterization with chronic urinary retention.

Patients' metabolic status and the extent of disease at presentation is an important factor in the prognosis of Fournier's gangrene [10].

Attempts to predict mortality in patients with Fournier's gangrene include the use of the Fournier gangrene severity index (FGSIS), and patient vital signs, and 
laboratory tests to calculate a score that could be used to monitor therapy and predict mortality [11]. FGSIS involves a determination of some laboratory parameters such as creatinine, bicarbonate, sodium, potassium, total protein, albumin, leukocyte count, hematocrit, lactate dehydrogenase, and alkaline phosphatase most of which could not be determined in our facility but did not serve as a hindrance to the treatment and survival of our patients.

The value of hyperbaric oxygenation in Fournier's gangrene remains unproven, but there are theoretical reasons why it may be beneficial, at least in some cases [6]. The mortality in Fournier's Gangrene is highest in patients presenting with sepsis, diabetes mellitus, and late admissions to the hospital [12]. Severe sepsis was a complication of both patients in this report.

Hospitalization for this disease is extremely long with a reported average of six weeks [13]. Our patients survived and were discharged 50 days and 47 days after admission to the hospital, despite the severity of their condition.

\section{Conclusions}

Fournier's gangrene is an uncommon but severe necrotizing infection of the external genitalia and perineum which can spread to involve the superficial fascia of the lower abdominal wall with rapid progression of gangrene and deterioration of the patient. Early recognition with aggressive surgical debridement, resuscitation, broad-spectrum antibiotics, and nutritional support remains the cornerstones of therapy.

Patients often may require care from a combined effort of urological and general surgeons; hence we suggest a multidisciplinary approach to improve patient outcomes not only in inpatient survival but also in the quality of life.

Clinical parameters should serve as a guide to the response of broad-spectrum antibiotics when there is limited capability to identify specific organisms implicated and their sensitivity pattern. Inability to carry out culture and sensitivity studies of the wound discharge should not impede good clinical management based on time-tested principles such as the use of broad-spectrum antibiotics, surgical debridement, and supportive care.

Repeated surgical debridement on the ward under analgesia and sedation may be required for complete eradication of infection.

\section{Statement of Informed Consent}

Verbal informed consent was obtained from the patients for the information and images used in this publication.

\section{Conflicts of Interest}

The authors declare no conflicts of interest regarding the publication of the paper.

\section{References}

[1] Marynowski, M.T. and Aronson, A.A. (2008) Fournier's Gangrene Medicine. 
https://scirp.org/reference/ReferencesPapers.aspx?ReferenceID=2166784

[2] Eke, N. (2000) Fournier's Gangrene: A Review of 1726 Cases. British Journal of Surgery, 87, 718-728. https://doi.org/10.1046/j.1365-2168.2000.01497.x

[3] Sorensen, M.D. and Krieger, J.N. (2016) Fournier's Gangrene: Epidemiology and Outcomes in the General US Population. Urologia internationalis, 97, 249-259. https://doi.org/10.1159/000445695

[4] Stone, H.H. and Martin Jr., J. (1972) Synergistic Necrotizing Cellulitis. Annals of Surgery, 175, 702-711. https://doi.org/10.1097/00000658-197205000-00010

[5] Papaconstantinou, I., Yiallourou, A.I., Dafnios, N., Grapsa, I., Polymeneas, G. and Voros, D. (2011) Successful Treatment of a Severe Case of Fournier's Gangrene Complicating a Perianal Abscess. Case Reports in Medicine, 2011, Article ID: 702429. https://doi.org/10.1155/2011/702429

[6] Laucks, S.S. (1994) Fournier's Gangrene. Surgical Clinics of North America, 74, 1339-1352. https://doi.org/10.1016/S0039-6109(16)46485-6

[7] Baddoe, E.A., Acheampong, F.Q. and da Rocha-Afodu, J.T. (2009) Principles and Practice of Surgery Including Pathology in the tropics. Assemblies of God Literature Centre Ltd., Accra.

[8] Villanueva-Sáenz, E., Hernández-Magro, P.M., Ovalle, M.V. and Vega, J.M. (2002) Experience in Management of Fournier's Gangrene. Techniques in Coloproctology, 6, 5-13. https://doi.org/10.1007/s101510200001

[9] Perry, T.L., Heyse, R.P., Little, A. and Johnson, M. (2010) Large Flap Preservation in a Patient with Extensive Necrotizing Fasciitis: Is Vertical Transmission of Infection Directly Proportional to Centrifugal Spread of Disease? Wounds. A Compendium of Clinical Research and Practice, 22, 146-150.

[10] Yeniyol, C.O., Suelozgen, T., Arslan, M. and Ayder, A.R. (2004) Fournier's Gangrene: Experience with 25 Patients and Use of Fournier's Gangrene Severity Index Score. Urology, 64, 218-222. https://doi.org/10.1016/j.urology.2004.03.049

[11] Sorensen, M.D., Krieger, J.N., Rivara, F.P., Klein, M.B. and Wessells, H. (2009) Fournier's Gangrene: Management and Mortality Predictors in a Population-Based Study. The Journal of Urology, 182, 2742-2747.

https://doi.org/10.1016/j.juro.2009.08.050

[12] Czymek, R., Hildebrand, P., Kleemann, M., Roblick, U., Hoffmann, M., Jungbluth, T., et al. (2009) New Insights into the Epidemiology and Etiology of Fournier's Gangrene: A Review of 33 Patients. Infection, 37, 306-312. https://doi.org/10.1007/s15010-008-8169-X

[13] Mindrup, S.R., Kealey, G.P. and Fallon, B. (2005) Hyperbaric Oxygen for the Treatment of Fournier's Gangrene. The Journal of Urology, 173, 1975-1977.

https://doi.org/10.1097/01.ju.0000158129.56571.05 